\title{
Regional and Hemispheric Asymmetries of Cerebral Hemodynamic and Oxygen Metabolism in Newborns
}

\author{
Pei-Yi Lin ${ }^{1,2}$, Nadège Roche-Labarbe ${ }^{1}$, Mathieu Dehaes ${ }^{3}$, Angela Fenoglio ${ }^{3}$, P. Ellen Grant ${ }^{3}$ and Maria Angela Franceschini $^{1}$ \\ ${ }^{1}$ The Optics Division, Athinoula A. Martinos Center for Biomedical Imaging, Massachusetts General Hospital, Harvard Medical \\ School, Charlestown, MA 02129, USA, ${ }^{2}$ Department of Biomedical Engineering, National Cheng Kung University, Tainan, 70101, \\ Taiwan and ${ }^{3}$ Fetal-Neonatal Neuroimaging and Developmental Science Center, Children's Hospital Boston, Harvard Medical School, \\ Boston, MA 02115, USA
}

Address correspondence to Maria Angela Franceschini, Athinoula A. Martinos Center for Biomedical Imaging, Massachusetts General Hospital, Bldg. 149, 13th Street, Charlestown, MA 02129, USA. Email: mari@nmr.mgh.harvard.edu.

\begin{abstract}
Understanding the evolution of regional and hemispheric asymmetries in the early stages of life is essential to the advancement of developmental neuroscience. By using 2 noninvasive optical methods, frequency-domain near-infrared spectroscopy and diffuse correlation spectroscopy, we measured cerebral hemoglobin oxygenation $\left(\mathrm{SO}_{2}\right)$, blood volume (CBV), an index of cerebral blood flow $\left(\mathrm{CBF}_{\mathrm{i}}\right)$, and the metabolic rate of oxygen $\left(\mathrm{CMRO}_{2 \mathrm{i}}\right)$ in the frontal, temporal, and parietal regions of 70 premature and term newborns. In concordance with results obtained using more invasive imaging modalities, we verified both hemodynamic $\left(\mathrm{CBV}, \mathrm{CBF}_{\mathrm{i}}\right.$, and $\left.\mathrm{SO}_{2}\right)$ and metabolic $\left(\mathrm{CMRO}_{2 \mathrm{i}}\right)$ parameters were greater in the temporal and parietal regions than in the frontal region and that these differences increased with age. In addition, we found that most parameters were significantly greater in the right hemisphere than in the left. Finally, in comparing age-matched males and females, we found that males had higher $\mathrm{CBF}_{\mathrm{i}}$ in most cortical regions, higher $\mathrm{CMRO}_{2 \mathrm{i}}$ in the frontal region, and more prominent right-left $\mathrm{CBF}_{\mathrm{i}}$ asymmetry. These results reveal, for the first time, that we can detect regional and hemispheric asymmetries in newborns using noninvasive optical techniques. Such a bedside screening tool may facilitate early detection of abnormalities and delays in maturation of specific cortical areas.
\end{abstract}

Keywords: brain development, cerebral asymmetry, diffuse correlation spectroscopy, near-infrared spectroscopy, newborns

\section{Introduction}

The adult cerebral cortex is highly variable across regions in both volume and sulcal depth and is asymmetric in both anatomical and functional aspects (Toga and Thompson 2003). Regional differences are present in adults, with brain volume in the frontal region greater than in the temporal, parietal, and occipital regions (Giedd et al. 1999). Males have larger brain volumes than females (Goldstein et al. 2001) but lower cerebral metabolism and blood flow (Baxter et al. 1987; Rodriguez et al. 1988; Gur RE and Gur RC 1990). The left hemisphere is typically dominant for language, handedness, and logical processing, while the right hemisphere is dominant for visuospatial processing, emotional perception, and musical ability (Sperry 1984; Borod 1992). Anatomical differences on magnetic resonance imaging (MRI) match this functional lateralization, with the sylvian fissure near the planum temporale generally deeper in the left than in the right hemisphere (Van Essen 2005) and the superior temporal sulcus distinctly deeper in the right than in the left. The right hemisphere is larger than the left in frontal, temporal, and parietal regions (Lancaster et al. 2003); the only exception is the occipital region, which is larger in the left than in the right hemisphere.

Several researchers have searched for the presence of anatomical and functional regional and hemispheric asymmetries in newborns, to determine whether such differences exist at birth. Regional differences in infants have been investigated using positron emission tomography (PET) and structural MRI. PET studies (Chugani et al. 1987; Kinnala et al. 1996; Chugani 1998) have shown that glucose uptake in the newborn brain is highest in the sensorimotor cortex with increases in parietal, temporal, and occipital regions during the first 3 months of life, consistent with improved function of visuospatial and visuosensorimotor integration at this age. Glucose uptake in the frontal cortex appears much later, at around 8 months of age. In agreement with glucose metabolism, synaptic density counts on ex vivo brain tissue show similar regional differences, with the auditory and parietal cortices reaching their highest level at age 3 months, whereas synapses in the prefrontal cortex continue to grow until 15 months of age (Huttenlocher and Dabholkar 1997). These functional regional differences are matched by anatomical differences as shown by recent structural MRI studies that report greater gray matter growth in the occipital and parietal regions than in the prefrontal region in newborns (Gilmore et al. 2007; Tzarouchi et al. 2009).

While regional differences between frontal and temporalparietal regions are large and verified across modalities, leftright hemispheric asymmetries in infants are more subtle, and results are not always in agreement. Hemispheric anatomical asymmetries of the fetal and premature cortex were first observed in postmortem studies, with the discovery that certain areas of the right hemisphere mature more quickly than corresponding areas in the left (Chi et al. 1977; Kretschmann et al. 1986; de Schonen and Mathivet 1989; Simonds and Scheibel 1989). While recent technological progress has allowed for the study of less compromised brains, confirmation of these early postmortem studies is not always convincing, with more recent MRI studies finding left-right hemispheric asymmetries in infants to be region specific (Tzarouchi et al. 2009; Dubois et al. 2010). In fact, contradictory results have been reported as to the size of left and right hemispheres. A larger left hemisphere has been observed on diagnostic ultrasound images in fetuses (Hering-Hanit et al. 2001) and on structural MRI volumetric images in newborns (Gilmore et al. 2007). A larger right hemisphere has been reported with ex vivo computed tomography scan in fetuses (de Schonen and Deruelle 1991) and with structural MRI in infants of different ages (Utsunomiya et al. 1999; Matsuzawa 
et al. 2001). Moreover, maturational differences have been reported within specific regions in the 2 hemispheres. For instance, while the superior temporal sulcus develops earlier in the right than in the left hemisphere (Dubois et al. 2008; Hill et al. 2010), the Broca's area and the planum temporale develop earlier in the left than in the right (Dubois et al. 2010). These areas, in addition to anatomical maturation, present early functional lateralization, with the right superior temporal sulcus specialized for the identification of faces and sounds (de Schonen and Deruelle 1991; Mento et al. 2010) and the Broca's area specialized for language processing (DehaeneLambertz et al. 2002; Peña et al. 2003).

Cortical asymmetry may also be influenced by genetic makeup (Annett 2002), as suggested by the differences in gene expression levels between the left and right hemispheres in human embryos (Sun et al. 2005, 2006). In particular, the higher expression of the transcription factor Lim Domain Only 4 (LMO4) in the right than in the left hemisphere may reflect either a differing topographic mapping in the 2 hemispheres or a difference in the time of cortical development, with the right hemisphere developing earlier than the left (Sun et al. 2005).

A few studies have reported sexual dimorphisms in infants, with males having larger cortical gray matter, white matter, and intracranial volume than females (Gilmore et al. 2007). Morphological hemispheric asymmetries between genders are not consistent with larger right-left brain volume asymmetry in females (Hering-Hanit et al. 2001) but are in accord with larger striate-extrastriate cortical volume asymmetry in males (De Lacoste et al. 1991).

These studies highlight the importance of characterizing regional and functional maturation during development, especially since a number of developmental disorders seem to be related to the abnormal development of the human cerebral cortex. In fact, abnormalities of cerebral cortical asymmetry and delays in maturation of specific cortical areas have been reported in neuropsychiatric disorders, such as schizophrenia, autism, and dyslexia (Falkai et al. 1992; Galaburda et al. 1994; Herbert et al. 2005).

Studies of brain development in large populations of neonates have not been performed because volumetric analysis of newborn brain MRIs is time intensive if small structures or regional segmentations are needed (Nishida et al. 2006). PET and single-photon emission computed tomography (SPECT) can be performed only on sick infants when clinically necessary, due to the radiation exposure required for these techniques. Electroencephalography or functional MRI functional studies yield information only on specifically stimulated cortical areas. In contrast, frequency-domain near-infrared spectroscopy (FDNIRS) and diffuse correlation spectroscopy (DCS) are noninvasive optical modalities that can measure cerebral vascular parameters, such as hemoglobin oxygenation $\left(\mathrm{SO}_{2}\right)$, blood volume (CBV), an index of cerebral blood flow $\left(\mathrm{CBF}_{\mathrm{i}}\right)$, and an index of the cerebral metabolic rate of oxygen $\left(\mathrm{CMRO}_{2 \mathrm{i}}\right)$ in infants (Franceschini et al. 2007; Roche-Labarbe et al. 2010). NIRS and DCS are particularly suitable in the neonatal population because they offer safe repeatable measurements at the bedside (Wolf and Greisen 2009) and take advantage of the infant's thin skull to measure cerebral hemoglobin parameters more accurately than could be done in adults (Dehaes et al. 2011). Moreover, regional and hemispheric maturation can be assessed more directly by estimating cerebral oxygen metabolism than by analysis of the size and depth of the cerebral cortex, by the response to a specific stimulation paradigm, or by the vascular development alone. Because FDNIRS and DCS use nonionizing radiation and can be performed at the bedside, a large population study in preterm infants is ethically feasible.

In a previous paper, we used FDNIRS and DCS to demonstrate that we can measure increases in $\mathrm{CMRO}_{2 \mathrm{i}}$ and $\mathrm{CBF}_{\mathrm{i}}$ with postmenstrual age (PMA) (Roche-Labarbe et al. 2011). Here, we test if we can measure regional and hemispheric differences in hemodynamic parameters and oxygen metabolism in the preterm and term infant cortex and determine whether these differences are influenced by gender and/or PMA.

\section{Materials and Methods}

\section{Subjects}

In this study, we recruited 55 preterm and 15 full-term neonates from the neonatal intensive care units and general nurseries of the Massachusetts General Hospital, Brigham and Women's Hospital, and Children's Hospital Boston between April 2008 and January 2011. We excluded infants with any of the following: germinal matrix hemorrhage (GMH) grade II or higher, intraventricular hemorrhage, parenchymal brain abnormalities on head ultrasound (HUS) or MRI, and 5-min Apgar $<7$. We included 4 premature infants with small unilateral or bilateral germinal matrix bleeds (grade I) (infants 17, 19, 23, and 40 in Roche-Labarbe et al. (2011)), which returned to normal according to HUS scans. The general clinical characteristics of the subjects are listed in Table 1 . The clinical summary for preterm infants is described in Roche-Labarbe et al. (2011) (infant 43 is excluded in this study because of bilateral germinal matrix and grade II intraventricular hemorrhage, and infant 42 because of 5-min Apgar of 5 due to lung prematurity). Infants were measured every 1-2 weeks during their hospital stay for a total of 244 measurements. The protocol was approved by our Institutional Review Board, and informed consent was obtained from all parents/guardians.

\section{Instrumentation}

We used a customized FDNIRS system (ISS Inc., Champaign, IL) to measure $\mathrm{SO}_{2}$ and $\mathrm{CBV}$ and a custom-built DCS system, similar to that developed by Drs Arjun Yodh and Turgut Durduran at the University of Pennsylvania (Cheung et al. 2001; Durduran et al. 2004), to estimate $\mathrm{CBF}_{\mathrm{i}}$. Technical details of the FDNIRS and DCS systems are described in Roche-Labarbe et al. (2010). In brief, the FDNIRS consisted of 16 laser sources divided into 2 identical groups of 8 lasers at different wavelengths in the near infrared, ranging from 660 to $830 \mathrm{~nm}$, and 2 photomultiplier tube detectors. Sources and detectors were coupled to fiber optics bundles (2.5 mm diameter) and arranged in a row on a black rubber probe $\left(5 \times 2 \times 1 \mathrm{~cm}^{3}\right)$ with source-detector distances of $1,1.5,2$, and $2.5 \mathrm{~cm}$ for infants $<37$ weeks PMA and $1.5,2,2.5$, and $3 \mathrm{~cm}$ for infants $>37$ weeks PMA (Fig. $1 a$ ). These distances are optimized for measuring neonatal cerebral cortex (Dehaes et al. 2011). The DCS consisted of one long coherence length laser source of $785 \mathrm{~nm}$ and 4 photon-counting avalanche photodiodes used as detectors. The DCS laser $(50 \mathrm{~mW}$ power) was coupled to a $62-\mu \mathrm{m}$ diameter multimode

\begin{tabular}{lc}
\hline Table $\mathbf{1}$ & \\
Clinical characteristics & $n=70$ \\
Clinical data & $31(24-42)$ \\
\hline Gestational age, wk, mean (range) & $1596(590-4281)$ \\
Birth weight, g, mean (range) & $38 / 32$ \\
Gender (M/F) & $3.6(0.2)$ \\
Postnatal age, wk, mean (SE) & $8.1(0.1)$ \\
Apgar score 5 min, mean (SE) &
\end{tabular}

Note: F: female; M: male; SE: standard error; wk: week. 


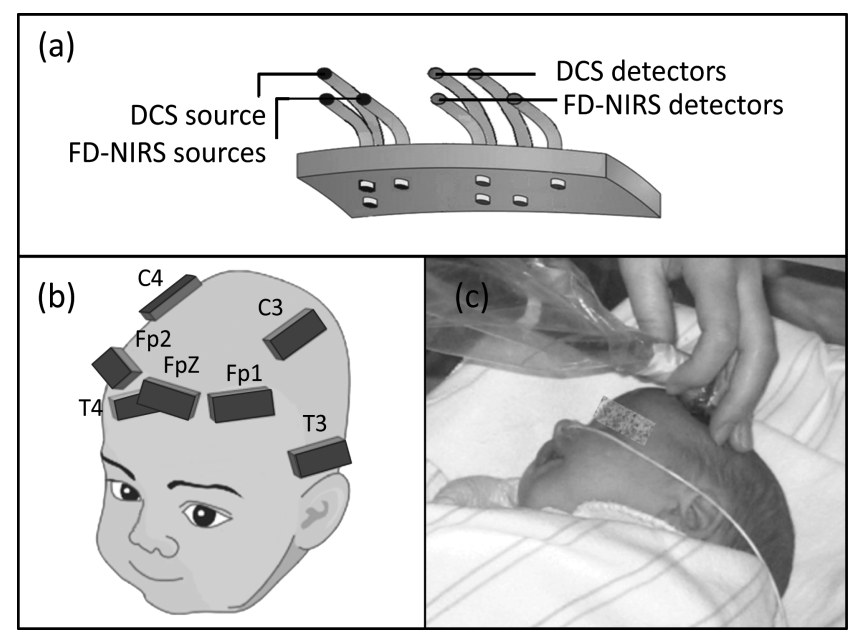

Figure 1. (a) The configuration of the probe. (b) Schematic drawing of the 7 measurement locations on the infant's head. (c) Picture of an infant during measurements.

fiber, and the detectors were coupled to 5.6- $\mu \mathrm{m}$ diameter single-mode optical fibers. Source and detector fibers were arranged on the probe in a row parallel to the FDNIRS fibers with source-detector distances of 1.5 ( 1 detector) and $2 \mathrm{~cm}$ ( 3 detectors). The intensity autocorrelation function of each detector channel was computed by a digital correlator (Correlator.com, Bridgewater, $\mathrm{NJ}$ ), and an autocorrelation curve over a delay time range of $200 \mathrm{~ns}$ to $0.5 \mathrm{~s}$ was acquired sequentially. In this study, only the DCS signals from the 3 detectors with a $2 \mathrm{~cm}$ separation were used for data analysis.

\section{Measurement Protocol}

Infants were measured at the bedside while resting between feeds. FDNIRS and DCS measurements were obtained in sequence from up to 7 locations (Fp1, FpZ, FP2, C3, C4, P3, P4, according to the 10-20 system; Fig. $1 b$ ) to cover the frontal, temporal, and parietal cortical regions. The optical probe was held in each location for 10-16 s of data acquisition (Fig. 1c). Measurements with each instrument were repeated up to 3 times in each location. Repositioning the probe in a slightly different area compensated for local inhomogeneities such as hair and superficial large vessels in order to ensure that the measurement was representative of the underlying brain region. The total examination time was $30-45 \mathrm{~min}$. Our decision to measure each location multiple times and the logistical need to keep the measurement time below 45 min prevented us from measuring more cerebral regions, such as the occipital area. Arterial oxygenation $\left(\mathrm{SaO}_{2}\right)$ was obtained from routine pulse oximeter monitors during the measurement session.

\section{Data Analysis}

The FDNIRS method used for $\mathrm{SO}_{2}$ and $\mathrm{CBV}$ quantification relies on measurements of the attenuation of radio frequency $(110 \mathrm{MHz})$ modulated light at several wavelengths. Light attenuation by biological tissues reflects contributions from both light absorption and scattering. By measuring not just light intensity attenuation but also phase delays at multiple distances, we can separate scattering and absorption coefficients, and from the absorption at multiple wavelengths, we can quantify hemoglobin concentration (Fantini et al. 1994). The accuracy of the FDNIRS method in recovering optical properties and hemoglobin parameters has been extensively tested in the past 18 years in both phantoms (Fishkin et al. 1995; Pogue et al. 2000) and animals (Hueber et al. 2001; Nelson et al. 2006) and has been validated and applied in humans (De Blasi et al. 1995; Franceschini et al. 1997; Choi et al. 2004; Zhao et al. 2005; Petrova and Mehta 2010).

DCS measures microvascular blood flow in deep tissue by quantifying the temporal intensity fluctuations of multiply scattered light that arise from Doppler shifts produced by moving red blood cells. The technique, introduced in the late 1990s (Boas et al. 1995), is similar to laser Doppler blood flowmetry (i.e., they are Fourier transform analogs), but light transmission through thick tissue results in low light levels that require photon counting detection techniques. Thus, DCS measures an autocorrelation function of the intensity fluctuations rather than a power spectrum. Blood flow changes measured with DCS have been extensively validated against other techniques in animal and human studies (Yu et al. 2005; Zhou et al. 2009; Carp et al. 2010; Durduran et al. 2010; Kim et al. 2010). More recently, our group and others have conducted animal and human (Yu et al. 2007; Buckley et al. 2009; Roche-Labarbe et al. 2010) validation studies to show how DCS provides an absolute number that scales with blood flow. By combining the FDNIRS measure of $\mathrm{SO}_{2}$ and the DCS measure of a $\mathrm{CBF}_{\mathrm{i}}$, we can estimate $\mathrm{CMRO}_{2 \mathrm{i}}$, as described in Roche-Labarbe et al. (2010).

FDNIRS and DCS raw data were post-processed using our standard automated data analysis tools developed in-house using MATLAB. For each subject, each measurement session, and each location, absorption coefficients measured with the FDNIRS were used to calculate both hemoglobin concentrations and blood flow index (Roche-Labarbe et al. 2010). An average of the scattering coefficients across the whole population was used in the calculations of $\mathrm{CBF}_{\mathrm{i}}$. The software also included data quality assessment and data rejection based on previously established statistical criteria (Roche-Labarbe et al. 2010). From the repeated measures in each location, we verified that reproducibility was consistent with previously reported values (Roche-Labarbe et al. 2010). Across all infants and all locations, we found the following coefficient of variation (standard deviation [SD]/mean): $4 \%$ for $\mathrm{SO}_{2}, 10 \%$ for hemoglobin concentration, and $13 \%$ for $\mathrm{CBF}_{\mathrm{i}}$.

To investigate regional differences, parietal $(P)$ values were obtained by averaging left and right parietal data, temporal $(T)$ values were obtained by averaging left and right temporal data, and frontal $(F)$ values were obtained by averaging middle, left, and right frontal data. To investigate hemispheric asymmetries, left hemisphere $(L)$ values were obtained by averaging left temporal and parietal data, while right hemisphere $(R)$ values were obtained by averaging right temporal and parietal data. We also compared all possible combinations of single locations to verify the consistency of the findings.

For the paired comparisons, we included subsets of the 70 infants and 244 sessions available that contained data points in the 2 locations compared. In $17 \%$ of the sessions, we did not acquire data in a complete set of 7 locations. Moreover, 34\% of the FDNIRS measurements and 28\% of the DCS measurements did not pass the data quality criteria. This left us with a total of 886 data points with estimated $\mathrm{CMRO}_{2 \mathrm{i}}\left(\mathrm{CMRO}_{2 \mathrm{i}}\right.$ is the parameter with the lowest number of data points being a combination of FDNIRS and DCS data), and an average of $103 \pm 33$ $\mathrm{CMRO}_{2 \mathrm{i}}$ paired points for the various comparisons. Using all of the available points led us to paired comparisons that included different infants and different numbers of data points. To ensure consistency, we ran paired comparisons in a complete subset of 36 measurement sessions from 21 infants for which we had all data points for all measured parameters in each location. In both cases-inclusion of all available data points or a complete subset-we performed paired $t$-tests for paired measurements (i.e., temporal vs. frontal, parietal vs. frontal, temporal vs. parietal, and left vs. right) in order to assess statistical significance of regional and hemispheric differences in each measured parameter.

To test the effect of PMA, we divided infants into 3 groups $(<32$, 32-36, and >36 weeks PMA) and performed factorial analysis of variance (ANOVA) to determine whether regional or hemispheric asymmetry was different as a function of PMA. We performed post hoc Bonferroni tests when the ANOVA showed significance.

For gender differences, we selected 22 age- and GA-matched males and females (Supplementary Table 1S) and performed $t$-tests for independent measurements to determine whether the vascular and metabolic parameters and regional and hemispheric asymmetries were significantly different between males and females.

We observed that in 161 measurement sessions (68\% of the sessions), infants were lying on their right side at presentation and were turned on the other side to complete measurements on all head locations. Infants began on their left side in only $32 \%$ of the measurements. Because of this large difference, we tested whether the side at 
presentation affected our findings. We divided the measurement sessions according to the side at presentation and compared each parameter between the 2 groups using a student $t$-test.

The statistical significance was set to $P<0.05$ for all of the tests.

\section{Results}

The scatterplots in Figure 2 show the regional and hemispheric differences in $\mathrm{CBF}_{\mathrm{i}}, \mathrm{SO}_{2}, \mathrm{CBV}$, and $\mathrm{CMRO}_{2 \mathrm{i}}$, including all available data points (the mean and SD of the measured parameters are listed in Supplementary Table 3S). Table 2 summarizes the regional and hemispheric comparison results by reporting percent differences and statistical significance. For the regional comparison, all measured parameters are greater in the temporal and parietal regions than in the frontal region. The largest differences are observed in $\mathrm{CBF}_{\mathrm{i}}$, which is $32 \%$ larger in the temporal than in the frontal region and $25 \%$ larger in the parietal than in the frontal region. The smallest differences, though still significant, are observed in $\mathrm{SO}_{2}$, which
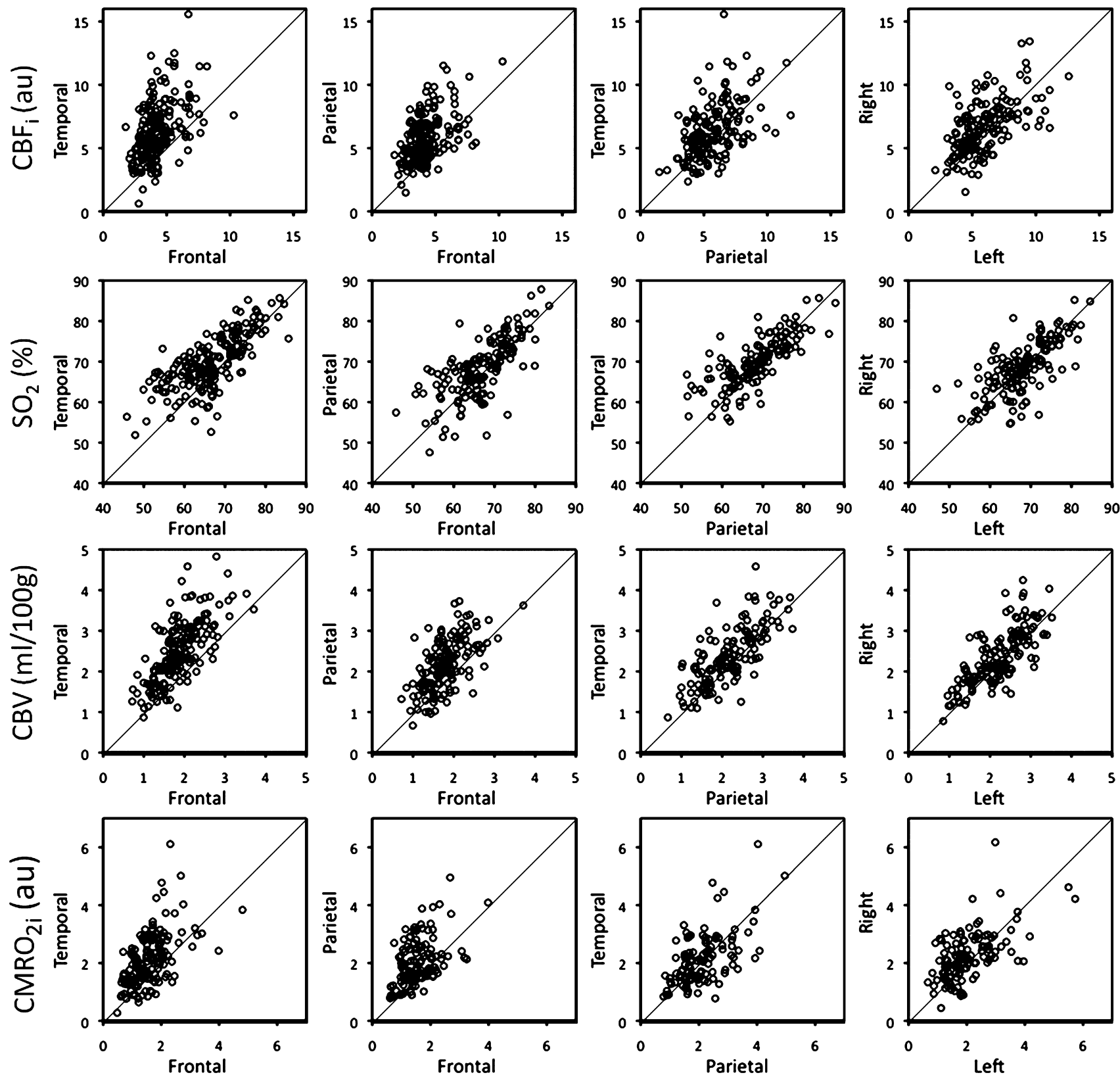

Figure 2. Scatterplots of $\mathrm{CBF}_{\mathrm{i}}, \mathrm{SO}_{2}, \mathrm{CBV}$, and $\mathrm{CMRO}_{2 \mathrm{i}}$ (row top to bottom) for temporal versus frontal, parietal versus frontal, and temporal versus parietal regions and right versus left hemispheres (columns left to right). The diagonal line marks the zero difference. 
is only $2-5 \%$ larger in the temporal and parietal than in the frontal region. We also observed significant differences in the vascular parameters between the temporal and parietal regions, with $\mathrm{CBF}_{\mathrm{i}}(10 \%), \mathrm{SO}_{2}(3 \%)$, and $\mathrm{CBV}(10 \%)$ larger in the temporal region than in the parietal region. In contrast, $\mathrm{CMRO}_{2 \mathrm{i}}$ did not differ between the temporal and parietal regions. For the hemispheric asymmetry analysis, $\mathrm{CBF}_{\mathrm{i}}(10 \%), \mathrm{CBV}(7 \%)$, and $\mathrm{CMRO}_{2 \mathrm{i}}(9 \%)$ are significantly larger in the right hemisphere than in the left.

We also performed paired analysis on the complete subset of 36 measurement sessions. The mean and SD of the measured parameters from the complete subset are listed in Table 3. The findings of regional difference and hemispheric asymmetry findings of this subset are consistent with those in the set including all available measurements, further validating the results (see Supplementary Table $2 S$ that summarizes the regional and hemispheric comparison results for the complete subset group).

The paired comparisons derived from comparing each of the 7 locations also confirmed the general trends revealed by the regional and hemispheric analysis. In some cases, statistical significance was not reached because of the lower number of measurements points when using single locations. For instance, $\mathrm{CMRO}_{2 \mathrm{i}}$ is larger in the right than in the left hemisphere, but while it reaches statistical significance in the parietal region (RP $>$ LP 14\%, $P<0.001$ ), it does not reach significance in the temporal and frontal regions (RT $>$ LT $4 \%, P=0.6$, and RF $>$ LF $3 \%, P=0.3)$.

The paired analysis of 21 measurements sessions from 4 infants with grade I germinal matrix bleeds showed the same regional and hemispheric asymmetries.

Blood flow and blood volume differences between the temporal and frontal and between the parietal and frontal regions increased with PMA and chronological age. Hemoglobin oxygenation differences between the temporal and parietal regions were significantly larger in the $>36$ weeks PMA group than in the 32-36 weeks PMA group. For $\mathrm{CMRO}_{2 \mathrm{i}}$, differences between regions did not increase with PMA. Also, for all parameters, no significant changes with PMA were observed in the differences between left and right hemispheres. Figure 3 shows the differences with PMA results in the cases where the post hoc test reached significance.

There is also evidence of sexual dimorphism in newborns. Males had significantly higher $\mathrm{CBF}_{\mathrm{i}}$ in most of the regions and significantly larger $\mathrm{CMRO}_{2 \mathrm{i}}$ in the frontal region compared with GA- and age-matched females (Fig. 4). Moreover, $\mathrm{CBF}_{\mathrm{i}}$ showed a more prominent right-left asymmetry $(P=0.001)$ in males than in females.

As for the side on which infants were lying at presentation, results showed no significant differences, with right hemisphere parameters larger than left independent of the side at

Table 3

Mean and SD of measured parameters from the subset of 36 complete sessions

\begin{tabular}{lrrrrr} 
& \multicolumn{1}{c}{ Parietal } & \multicolumn{1}{c}{ Temporal } & \multicolumn{1}{c}{ Frontal } & \multicolumn{1}{c}{ Right } & \multicolumn{1}{c}{ Left } \\
\hline $\mathrm{CBF}_{\mathrm{i}}(\mathrm{au})$ & $6.0(1.8)$ & $6.5(1.9)$ & $4.1(0.9)$ & $6.9(1.7)$ & $5.7(1.8)$ \\
$\mathrm{SO}_{2}(\%)$ & $69.2(6.7)$ & $70.1(5.3)$ & $67.2(6.8)$ & $70.2(5.2)$ & $69.5(6.7)$ \\
$\mathrm{CBV}_{(\mathrm{mL} / 100 \mathrm{~g})}$ & $1.9(0.5)$ & $2.1(0.5)$ & $1.7(0.4)$ & $2.2(0.5)$ & $2.0(0.5)$ \\
$\mathrm{CMRO}_{2 \mathrm{i}}(\mathrm{au})$ & $2.1(0.8)$ & $2.1(0.8)$ & $1.5(0.4)$ & $2.3(0.7)$ & $2.0(0.9)$ \\
\hline
\end{tabular}

Note: The data are presented as mean (SD). presentation. This observation confirmed that the hemispheric asymmetry is not influenced by the side at presentation.

\section{Discussion}

For the first time, we were able to show regional and hemispheric differences in hemodynamic $\left(\mathrm{SO}_{2}, \mathrm{CBV}\right.$, and $\left.\mathrm{CBF}_{\mathrm{i}}\right)$ and oxygen metabolism $\left(\mathrm{CMRO}_{2 \mathrm{i}}\right)$ in the neonatal cerebral cortex using FDNIRS and DCS.

We found that all hemodynamic parameters and oxygen metabolism significantly were higher in the temporal and parietal cortical regions than in the frontal region in premature and term infants. The regional differences in oxygen metabolism are consistent with fluorodeoxyglucose PET findings that the primary sensory areas exhibit the highest glucose uptake in newborns (Chugani et al. 1987; Kinnala et al. 1996; Chugani 1998). Based on these PET studies, glucose utilization increases in the frontal cortex only between the ages of 6 and 12 months. In addition, the higher cerebral blood volume and blood flow in the temporal and parietal regions than in the frontal region are consistent with findings from previous NIRS studies (Keel et al. 1999) and SPECT (Younkin et al. 1988; Chiron et al. 1992; Børch and Greisen 1998). Using a simple NIRS system to measure 10 premature infants, Keel et al. (1999) found that hemoglobin concentration increased $40-50 \%$ from the frontal to lateral regions. Børch and Greisen (1998) used SPECT to measure blood flow in 12 preterm infants and found higher flow in the motor area than in the frontal and occipital areas. Chiron et al. (1992) measured regional cerebral blood flow with SPECT in 42 children between 2 days and 19 years old. Cerebral blood flow was lower at birth than in adults, and the time needed to reach adult values differed for each cortical region. The shortest time was in the primary cortex and the longest in the associative cortex. They concluded that changes in blood flow, like changes in metabolism, should be related to development of cognitive functions of the corresponding region. The regional differences we measured in hemodynamic and oxygen metabolism in premature and term infants are consistent with cognitive, behavioral, and functional developmental differences (Zeanah et al. 2008) but also with finding related to dendritic and axonal growth (Schade and Van Groenigen 1961), white matter myelination (Ferrie et al. 1999), brain morphology, and synaptogenesis, all of which start in primary sensory areas and evolve with age to the prefrontal cortex.

We also found hemoglobin oxygenation differences between the temporal-parietal and frontal regions, with higher $\mathrm{SO}_{2}$ in areas of higher functional activity. These differences, even if significant, were small, in agreement with our previous findings of smaller variance in $\mathrm{SO}_{2}$ with age and disease than in $\mathrm{CBV}$, $\mathrm{CBF}_{\mathrm{i}}$, and $\mathrm{CMRO}_{2}$ (Takahashi et al. 1999; Franceschini et al. 2007; Grant et al. 2009; Boas and Franceschini 2011).

The differences between the temporal-parietal and frontal regions seemed to increase with PMA, with larger differences in infants $>36$ weeks PMA than in infants $<32$ weeks PMA. This was consistent with the results of Huttenlocher and Dabholkar (1997), which showed that synaptic density increases more rapidly in the auditory cortex, where the synapse number peaks at 3 months of age. It takes 3.5 years for the prefrontal cortex to reach the same number of synapses as the auditory cortex (Huttenlocher and Dabholkar 1997). Also, structural MRI studies have shown that in the first 2 months of life, gray 

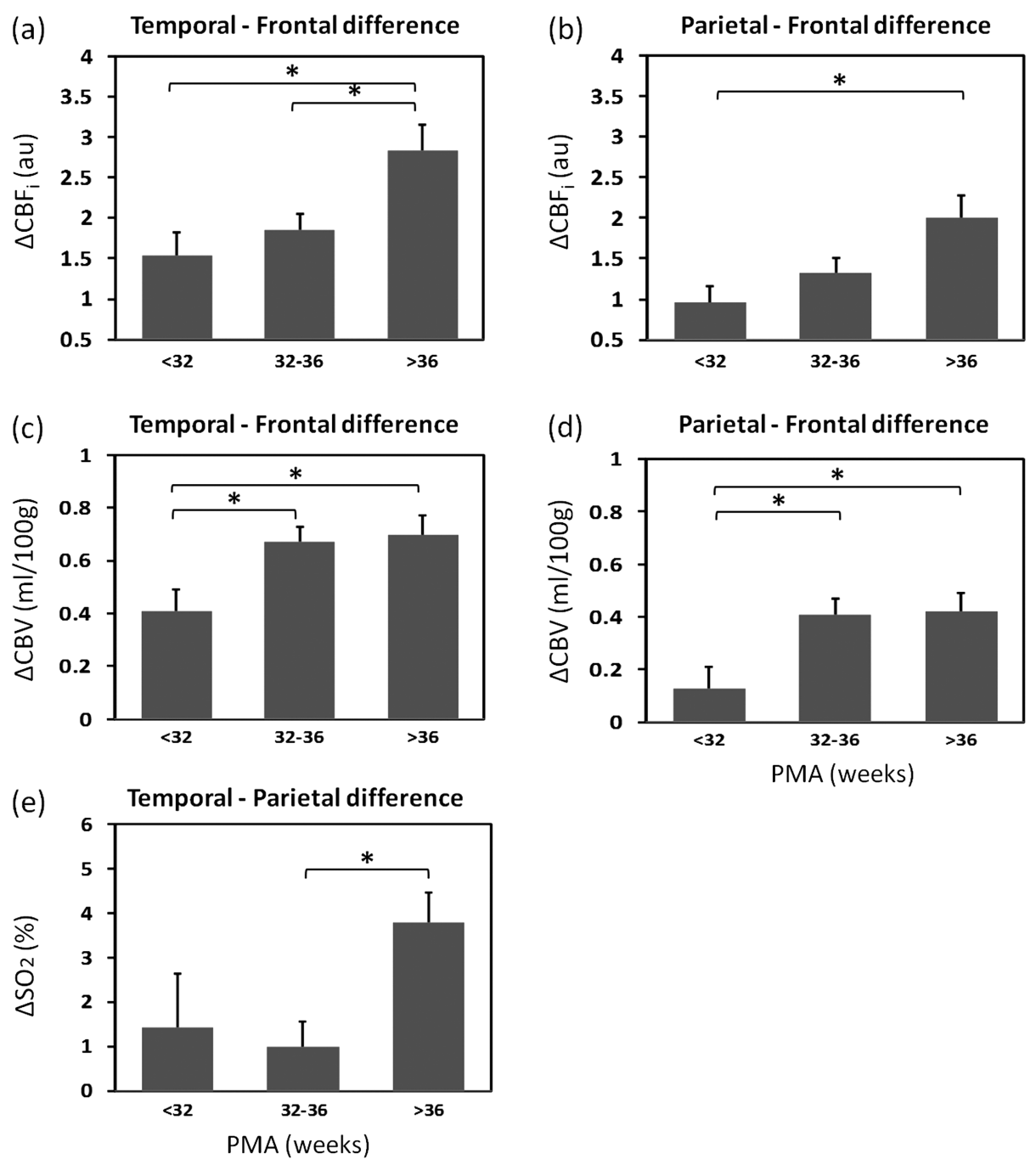

Figure 3. Temporal-frontal difference and parietal-frontal difference in $\mathrm{CBF}_{i}(a$ and $b)$ and $\mathrm{CBV}(c$ and $d)$, and temporal-parietal difference in $\mathrm{SO}_{2}(e)$ for the $3 \mathrm{PMA}$ groups. Mean differences and one standard error are reported and *indicates statistically significant differences between groups $(P<0.05$ in post hoc test).
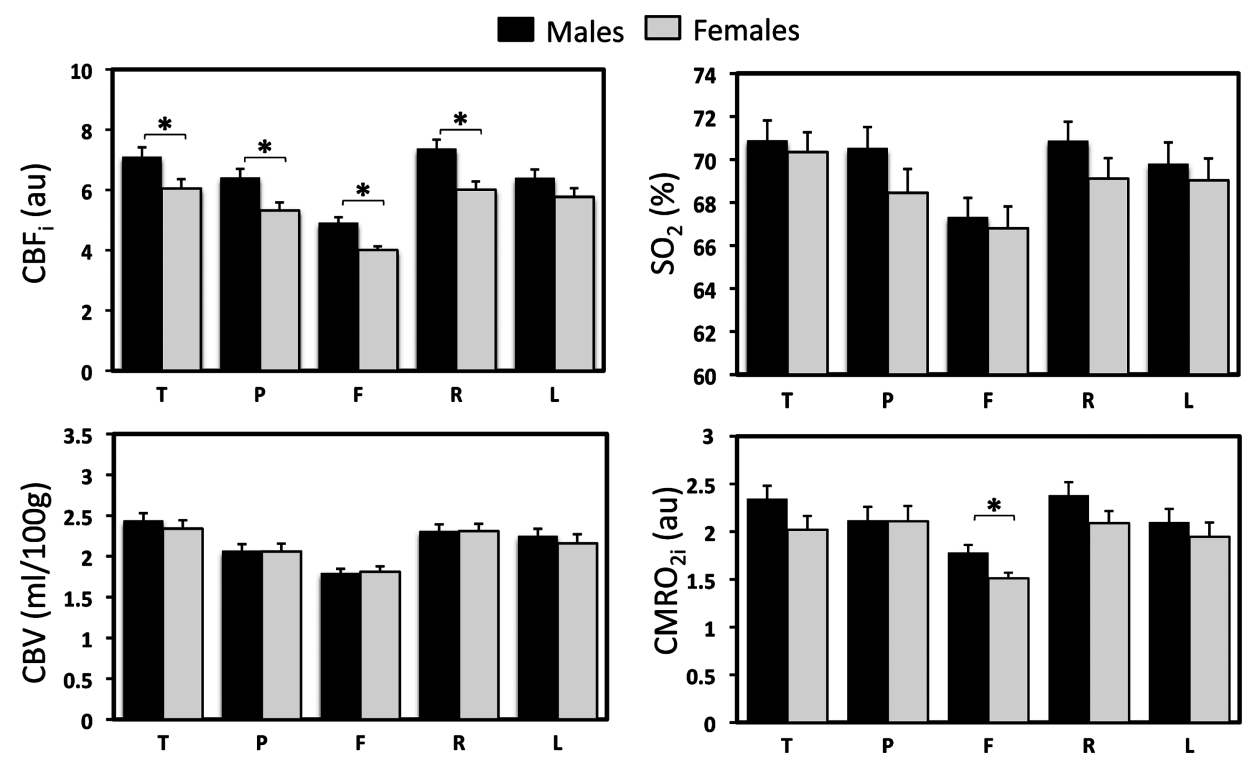

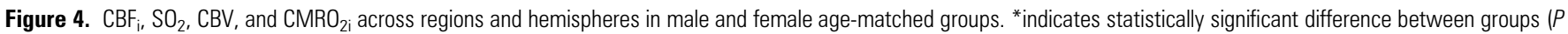
$<0.05$ in student $t$-test). $T$, temporal; $P$, parietal; $F$, frontal; $R$, right; and $L$, left. 
matter grows faster in the parietal and occipital regions than in the frontal and prefrontal regions (Gilmore et al. 2007; Tzarouchi et al. 2009).

When comparing the temporal and parietal regions, we found higher $\mathrm{CBF}_{\mathrm{i}}, \mathrm{CBV}$, and $\mathrm{SO}_{2}$ in the former. Differences were not as large as between the temporal and frontal or the parietal and frontal regions but were still statistically significant. Our finding of higher CBF in the temporal than in the parietal cortex is in agreement with results from SPECT measurements in premature (Younkin et al. 1988) and older infants (Chiron et al. 1992). However, we did not measure any significant difference in $\mathrm{CMRO}_{2 \mathrm{i}}$ between these 2 regions. The temporalparietal differences found in hemodynamic parameters but not in $\mathrm{CMRO}_{2}$ may be due to differences in microvasculature in these 2 regions, which will affect hemoglobin parameters but not necessarily metabolism. On the contrary, Chugani et al. found that glucose metabolism was higher in the sensorimotor areas than in the temporal areas in newborns (Chugani et al. 1987; Kinnala et al. 1996; Chugani 1998). This divergence may be due to the difference in gestational age between our population and the PET study population, or perhaps to the health conditions and/or the small number of infants enrolled in the PET study, or the mismatch in measurement locations between our study and the PET studies.

Another relatively small but important difference was between the left and right hemisphere. $\mathrm{CBV}, \mathrm{CBF}_{\mathrm{i}}$, and $\mathrm{CMRO}_{2 \mathrm{i}}$ were consistently higher in the right temporal and parietal regions than in the left while $\mathrm{SO}_{2}$ differences did not reach significance. As discussed in the Introduction, left-right brain differences during development have been investigated with many techniques, but no clear answer has yet been found. This is in part due to the fact that the differences are small (Børch and Greisen 1998), within the SNR of the technologies used, and may need a large population to reach significance. This is not always feasible in healthy infant populations with techniques, such as PET and MRI. Our safe noninvasive bedside approach allows us to measure a large number of infants while our automated rejection criteria enables us to discard bad data sets, reducing variance and increasing sensitivity. As a result, we could detect a $7-10 \%$ difference $(P<0.05)$ in blood flow, blood volume, and oxygen consumption between the left and right hemispheres. Our findings of right hemisphere dominance are in agreement with the "Right-hemisphere conservatism" theory (Geschwind and Galaburda 1985). Because the right hemisphere develops earlier, its development is less subject to external influences than that of the left hemisphere. As a result, there is greater uniformity across individuals in those functions for which the right hemisphere is dominant. The delay in maturation of the left hemisphere may allow for a higher plasticity with functional stimulation, such as language exposure and motor movements (Dubois et al. 2008). Moreover, in humans, as in animals, the right hemisphere sustains those functions necessary to the survival of the species, including visuospatial or emotional processes, which accounts for its earlier development (Geschwind and Galaburda 1985). Compelling support for this theory has been provided by Sun et al. (2005, 2006), who found significant asymmetries of gene expression in embryos as early as 12 weeks gestational age. In particular, the transcription factor LMO4 gene is consistently more highly expressed in the right perisylvian cortex than in the left. LMO4 relates to the regulation of normal sensorimotor control (Huang et al.
2009), and its delayed expression in the left hemisphere may be responsible for the left lateralization of the sensorimotor control.

Several studies have reported earlier development of Broca's area and the planum temporale in the left than the right (Dubois et al. 2008, 2010; Hill et al. 2010). The location of our probe was more posterior, above the auditory cortex, and therefore, we have no measures of the left-right asymmetry of Broca's area and the planum temporale. Higher metabolism in the right temporal cortex is consistent with higher electrophysiological activity in the right for both pitch detection and discrimination measured in healthy preterm newborns (Mento et al. 2010).

Another interesting result from these measurements is the consistently higher cerebral blood flow in newborn males than females in most cortical regions. Baenziger et al. (1994) also reported higher $\mathrm{CBF}$ in male than female infants. Using SPECT, they determined that premature males have significantly higher CBF than females. As in their study, the gender differences we found cannot be attributed to differences in physiologic parameters or activation state, which were similar in the 2 groups. The difference is reversed in adults, with cerebral blood flow higher in females than in males (Gur et al. 1982; Rodriguez et al. 1988; Gur RE and Gur RC 1990). The higher blood flow that we measured in male premature and term newborns is of particular interest because male infants are more at risk for disability, handicap, and mortality than females (Brothwood et al. 1986; Elsmén et al. 2004; Mohamed and Aly 2010). In addition to higher blood flow, when comparing for gender differences, we found larger right-left asymmetry of $\mathrm{CBF}_{\mathrm{i}}$ in males than in females. No blood flow hemispheric asymmetry differences with gender have been reported in the literature. Morphological hemispheric asymmetries that are larger in males than in females have been reported in the past (De Lacoste et al. 1991), and it has been suggested that they may be associated with differences in fetal testosterone levels affecting the development of spatial ability (Geschwind and Galaburda 1985). However, the conflicting result has been reported in morphological hemispheric differences between males and females (Gilmore et al. 2007).

In this study, we included very premature infants with no indication of brain injury on HUS or MRI and with normal neurological follow-up exams. Of these very premature infants, 4 had grade I GMH. Cerebral blood volume in these infants was significantly lower than in age- and GA-matched premature infants without grade I hemorrhage, but there was no significant differences in $\mathrm{CBFi}$ or $\mathrm{CMRO} 2 \mathrm{i}$. The reason for the decreased $\mathrm{CBV}$ is unclear. However, we verified that the regional and hemispheric asymmetries were maintained in these 4 infants. This may be not the case when more extensive brain injuries are present, and altered asymmetries may provide additional diagnostic tools in addition to the detection of abnormal oxygen metabolism and vascular parameters with injury (Grant et al. 2009; Verhagen et al. 2010).

Since some of the regional differences we found are very subtle and within measurement errors, technological improvements are needed if this is to become a clinical tool to reliably detect asymmetries in individual subjects. Measurement reproducibility can be improved by implementing feedback on the quality of the coupling and the pressure between the optical probe and head surface. The use of more sophisticated mathematical models that take into account the head geometry 
will further reduce errors. The availability of additional channels will also improve data modeling and reduce errors.

In addition to reducing measurement errors, implementation of real-time feedback on data quality will guide repeated measures to reduce the frequency of discarded measures. Finally, full integration of the FDNIRS and DCS systems will reduce acquisition time, by either reducing time at the bedside or allowing monitoring of additional brain regions.

\section{Conclusion}

Based on measurements with FDNIRS and DCS, we have provided for the first time a quantitative description of hemispheric asymmetries and regional differences in cerebral oxygen metabolism, cerebral blood flow, and cerebral blood volume in premature and term newborns. We found regional differences consistent with PET studies, with metabolism substantially higher in the temporal and parietal than in the frontal region. We also found metabolism higher in the right than in the left hemisphere. This supports current theories about the delay in maturation of the left hemisphere in newborns allowing for higher plasticity with environmental stimulation, such as language exposure and motor skills. We observed consistently higher $\mathrm{CBF}_{\mathrm{i}}$ in males than in females, which may be related to the greater vulnerability of newborn males with respect to females.

Establishing the baseline of hemispheric and regional differences in preterm and term neonates will be valuable in determining regional and functional maturation during development, especially as a number of developmental disorders, including schizophrenia, autism, and dyslexia, seem to be related to the abnormal development of human cerebral cortex.

\section{Supplementary Material}

Supplementary material can be found at: http://www.cercor. oxfordjournals.org/

\section{Funding}

National Institute of Health (R01-HD042908, R21-HD058725, and P41-RR14075) and National Science council of Republic of China (NSC 100-2321-B-006 -018 to P.-Y. L.)

\section{Notes}

We thank Marcia Kocienski-Filip for her assistance during measurements, which is supported by the Clinical Translational Science Award UL1RR025758 to Harvard University and Brigham and Women's Hospital from the National Center for Research Resources. We thank Bruce Rosen, David Boas, Maddy Artunduaga, Alpna Aggarwal, Sarah M. Barnett, Kalpathy S. Krishnamoorthy, Linda J. Van Marter, Robert M. Insoft, Jonathan $\mathrm{H}$. Cronin, Elizabeth A. Warren, and the neonatal intensive care units staff for help and support on the project. We thank Dr George Cowan for guidance in pursuing this project. We also thank Gary Boas for editing the manuscript. The content is solely the responsibility of the authors and does not necessarily represent the official views of the National Center for Research Resources or the National Institutes of Health. Conflict of Interest: None declared.

\section{References}

Annett M. 2002. Handedness and brain asymmetry: the right shift theory. New York: Taylor \& Francis Inc.

Baenziger O, Jaggi JL, Mueller AC, Morales CG, Lipp HP, Lipp AE, Duc G, Bucher HU. 1994. Cerebral blood flow in preterm infants affected by sex, mechanical ventilation, and intrauterine growth. Pediatr Neurol. 11:319-324.

Baxter LR Jr, Mazziotta JC, Phelps ME, Selin CE, Guze BH, Fairbanks L. 1987. Cerebral glucose metabolic rates in normal human females versus normal males. Psychiatry Res. 21:237-245.

Boas D, Campbell L, Yodh A. 1995. Scattering and imaging with diffusing temporal field correlations. Phys Rev Lett. 75:1855-1858.

Boas DA, Franceschini MA. Forthcoming 2011. Haemoglobin oxygen saturation as a biomarker: the problem and a solution. Philos Trans $\mathrm{R}$ Soc A. 369:4407-4424.

Børch K, Greisen G. 1998. Blood flow distribution in the normal human preterm brain. Pediatr Res. 43:28.

Borod JC. 1992. Interhemispheric and intrahemispheric control of emotion: a focus on unilateral brain damage. J Consult Clin Psychol. 60:339-348.

Brothwood M, Wolke D, Gamsu H, Benson J, Cooper D. 1986. Prognosis of the very low birthweight baby in relation to gender. Arch Dis Child. 61:559-564.

Buckley EM, Cook NM, Durduran T, Kim MN, Zhou C, Choe R, Yu G, Shultz S, Sehgal CM, Licht DJ, et al. 2009. Cerebral hemodynamics in preterm infants during positional intervention measured with diffuse correlation spectroscopy and transcranial Doppler ultrasound. Opt Express. 17:12571-12581.

Carp SA, Dai GP, Boas DA, Franceschini MA, Kim YR. 2010. Validation of diffuse correlation spectroscopy measurements of rodent cerebral blood flow with simultaneous arterial spin labeling MRI; towards MRI-optical continuous cerebral metabolic monitoring. Biomed Opt Express. 1:553-565.

Cheung C, Culver JP, Kasushi T, Greenberg JH, Yodh AG. 2001. In vivo cerebrovascular measurement combining diffuse near-infrared absorption and correlation spectroscopies. Phys Med Biol. 46:2053-2065.

Chi JG, Dooling EC, Gilles FH. 1977. Left-right asymmetries of the temporal speech areas of the human fetus. Arch Neurol. 34:346-348.

Chiron C, Raynaud C, Mazière B, Zilbovicius M, Laflamme L, Masure MC, Dulac O, Bourguignon M, Syrota A. 1992. Changes in regional cerebral blood flow during brain maturation in children and adolescents. J Nucl Med. 33:696-703.

Choi JH, Wolf M, Toronov V, Wolf U, Polzonetti C, Hueber D, Safonova LP, Gupta R, Michalos A, Mantulin W. 2004. Noninvasive determination of the optical properties of adult brain: near-infrared spectroscopy approach. J Biomed Opt. 9:221-229.

Chugani HT. 1998. A critical period of brain development: studies of cerebral glucose utilization with PET. Prev Med. 27:184-188.

Chugani HT, Phelps M, Mazziotta J. 1987. Positron emission tomography study of human brain functional development. Ann Neurol. 22:487-497.

De Blasi R, Fantini S, Franceschini M, Ferrari M, Gratton E. 1995. Cerebral and muscle oxygen saturation measurement by frequencydomain near-infra-red spectrometer. Med Biol Eng Comput. 33:228-230.

Dehaene-Lambertz G, Dehaene S, Hertz-Pannier L. 2002. Functional neuroimaging of speech perception in infants. Science. 298:2013-2015.

Dehaes M, Grant PE, Sliva D, Roche-Labarbe N, Pienaar R, Boas D, Franceschini M, Selb J. 2011. Assessment of the frequency-domain multi-distance method to evaluate the brain optical properties: Monte Carlo simulations from neonate to adult. Biomed Opt Exp. 2:552-567.

De Lacoste MC, Horvath D, Woodward D. 1991. Possible sex differences in the developing human fetal brain. J Clin Exp Neuropsychol. 13:831-846.

de Schonen S, Deruelle C. 1991. Visual field asymmetries for pattern processing are present in infancy. A comment on T. Hatta's study on children's performances. Neuropsychologia. 29:335-337.

de Schonen S, Mathivet E. 1989. First come, first served: a scenario about the development of hemispheric specialization in face recognition during infancy. Curr Psychol Cogn. 9:3-44.

Dubois J, Benders M, Cachia A, Lazeyras F, Ha-Vinh Leuchter R, Sizonenko SV, Borradori-Tolsa C, Mangin J, Hüppi PS. 2008. Mapping 
the early cortical folding process in the preterm newborn brain. Cereb Cortex. 18:1444-1454.

Dubois J, Benders M, Lazeyras F, Borradori-Tolsa C, Leuchter R, Mangin J, Hüppi P. 2010. Structural asymmetries of perisylvian regions in the preterm newborn. Neuroimage. 52:32-42.

Durduran T, Yu G, Burnett MG, Detre JA, Greenberg JH, Wang J, Zhou C, Yodh AG. 2004. Diffuse optical measurement of blood flow, blood oxygenation, and metabolism in a human brain during sensorimotor cortex activation. Opt Lett. 29:1766-1768.

Durduran T, Zhou C, Buckley EM, Kim MN, Yu G, Choe R, Gaynor JW, Spray TL, Durning SM, Mason SE, et al. 2010. Optical measurement of cerebral hemodynamics and oxygen metabolism in neonates with congenital heart defects. J Biomed Opt. 15:037004

Elsmén E, Steen M, Hellstrom-Westas L. 2004. Sex and gender differences in newborn infants: why are boys at increased risk? J Men's Health Gender. 1:303-311.

Falkai P, Bogerts B, Greve B, Pfeiffer U, Machus B, Folsch-Reetz B, Majtenyi C, Ovary I. 1992. Loss of sylvian fissure asymmetry in schizophrenia: a quantitative post mortem study. Schizophr Res. 7:23-32.

Fantini S, Franceschini MA, Gratton E. 1994. Semi-infinite-geometry boundary problem for light migration in highly scattering media: a frequency-domain study in the diffusion approximation. J Opt Soc Am B. 11:2128-2138.

Ferrie J, Barantin L, Saliba E, Akoka S, Tranquart F, Sirinelli D, Pourcelot L. 1999. MR assessment of the brain maturation during the perinatal period: quantitative T2 MR study in premature newborns. Magn Reson Imaging. 17:1275-1288.

Fishkin JB, So PT, Cerussi AE, Fantini S, Franceschini MA, Gratton E 1995. Frequency-domain method for measuring spectral properties in multiple-scattering media: methemoglobin absorption spectrum in a tissuelike phantom. Appl Opt. 34:1143-1155.

Franceschini MA, Moesta KT, Fantini S, Gaida G, Gratton E, Jess H, Mantulin WW, Seeber M, Schlag PM, Kaschke M. 1997. Frequencydomain techniques enhance optical mammography: initial clinical results. Proc Natl Acad Sci U S A. 94:6468-6473.

Franceschini MA, Thaker S, Themelis G, Krishnamoorthy KK, Bortfeld H, Diamond SG, Boas DA, Arvin K, Grant PE. 2007. Assessment of infant brain development with frequency-domain near-infrared spectroscopy. Pediatr Res. 61:546-551.

Galaburda AM, Menard MT, Rosen GD. 1994. Evidence for aberrant auditory anatomy in developmental dyslexia. Proc Natl Acad Sci U S A. 91:8010-8013.

Geschwind N, Galaburda AM. 1985. Cerebral lateralization: biological mechanisms, associations, and pathology: III. A hypothesis and a program for research. Arch Neurol. 42:634-654.

Giedd JN, Blumenthal J, Jeffries NO, Castellanos FX, Liu H, Zijdenbos A Paus T, Evans AC, Rapoport JL. 1999. Brain development during childhood and adolescence: a longitudinal MRI study. Nat Neurosci. 2:861-862.

Gilmore JH, Lin W, Prastawa MW, Looney CB, Vetsa YSK, Knickmeyer RC, Evans DD, Smith JK, Hamer RM, Lieberman JA. 2007. Regional gray matter growth, sexual dimorphism, and cerebral asymmetry in the neonatal brain. J Neurosci. 27:1255-1260.

Goldstein JM, Seidman LJ, Horton NJ, Makris N, Kennedy DN, Caviness VS, Faraone SV, Tsuang MT. 2001. Normal sexual dimorphism of the adult human brain assessed by in vivo magnetic resonance imaging. Cereb Cortex. 11:490-497.

Grant PE, Roche-Labarbe N, Surova A, Themelis G, Selb J, Warren EK, Krishnamoorthy KS, Boas DA, Franceschini MA. 2009. Increased cerebral blood volume and oxygen consumption in neonatal brain injury. J Cereb Blood Flow Metab. 29:1704-1713.

Gur RC, Gur RE, Obrist WD, Hungerbuhler JP, Younkin D, Rosen AD, Skolnick BE, Reivich M. 1982. Sex and handedness differences in cerebral blood flow during rest and cognitive activity. Science. 217:659-661.

Gur RE, Gur RC. 1990. Gender differences in regional cerebral blood flow. Schizophr Bull. 16:247-254.

Herbert MR, Ziegler D, Deutsch C, O'Brien L, Kennedy D, Filipek P, Bakardjiev A, Hodgson J, Takeoka M, Makris N. 2005. Brain asymmetries in autism and developmental language disorder: a nested whole-brain analysis. Brain. 128:213-226.

Hering-Hanit R, Achiron R, Lipitz S, Achiron A. 2001. Asymmetry of fetal cerebral hemispheres: in utero ultrasound study. Arch Dis Child Fetal Neonatal Ed. 85:F194-F196.

Hill J, Dierker D, Neil J, Inder T, Knutsen A, Harwell J, Coalson T, Van Essen D. 2010. A surface-based analysis of hemispheric asymmetries and folding of cerebral cortex in term-born human infants. J Neurosci. 30:2268-2276.

Huang Z, Kawase-Koga Y, Zhang S, Visvader J, Toth M, Walsh CA, Sun T. 2009. Transcription factor Lmo4 defines the shape of functional areas in developing cortices and regulates sensorimotor control. Dev Biol. 327:132-142.

Hueber D, Franceschini M, Ma H, Zhang Q, Ballesteros J, Fantini S, Wallace D, Ntziachristos V, Chance B. 2001. Non-invasive and quantitative near-infrared haemoglobin spectrometry in the piglet brain during hypoxic stress, using a frequency-domain multidistance instrument. Phys Med Biol. 46:41-62.

Huttenlocher PR, Dabholkar AS. 1997. Regional differences in synaptogenesis in human cerebral cortex. J Comp Neurol. 387:167-178.

Keel M, Wolf M, Baenziger O, Dietz V, von Siebenthal K, Bucher HU. 1999. Regional differences of cerebral hemoglobin concentration in preterm infants measured by near infrared spectrophotometry. Technol Health Care. 7:63-73.

Kim MN, Durduran T, Edlow BL, Buckley EM, Mesquita RC, Grady MS, Levine JM, Greenberg JH, Detre JA, Yodh AG. 2010. Diffuse optical measurements of cerebral blood flow and blood oxygenation during head elevation in healthy and brain-injured adults. In: Biomedical optics. Miami (Florida): OSA Technical Digest. p. BWF6.

Kinnala A, Suhonen-Polvi $H$, Aärimaa $T$, Kero $P$, Korvenranta $H$, Ruotsalainen U, Bergman J, Haaparanta M, Solin O, Nuutila P. 1996. Cerebral metabolic rate for glucose during the first six months of life: an FDG positron emission tomography study. Arch Dis Child Fetal Neonatal Ed. 74:F153-F157.

Kretschmann HJ, Kammradt G, Krauthausen I, Sauer B, Wingert F. 1986. Brain growth in man. Bibl Anat. 28:1-26.

Lancaster JL, Kochunov PV, Thompson PM, Toga AW, Fox PT. 2003. Asymmetry of the brain surface from deformation field analysis. Hum Brain Mapp. 19:79-89.

Matsuzawa J, Matsui M, Konishi T, Noguchi K, Gur RC, Bilker W, Miyawaki T. 2001. Age-related volumetric changes of brain gray and white matter in healthy infants and children. Cereb Cortex. 11:335-342.

Mento G, Suppiej A, Altoè G, Bisiacchi PS. 2010. Functional hemispheric asymmetries in humans: electrophysiological evidence from preterm infants. Eur J Neurosci. 31:565-574.

Mohamed MA, Aly H. 2010. Male gender is associated with intraventricular hemorrhage. Pediatrics. 125:e333-e339.

Nelson LA, McCann JC, Loepke AW, Wu J, Dor BB, Kurth CD. 2006 Development and validation of a multiwavelength spatial domain near-infrared oximeter to detect cerebral hypoxia-ischemia. J Biomed Opt. 11:064022.

Nishida M, Makris N, Kennedy DN, Vangel M, Fischl B, Krishnamoorthy KS, Caviness VS, Grant PE. 2006. Detailed semiautomated MRI based morphometry of the neonatal brain: preliminary results. Neuroimage. 32:1041-1049.

Peña M, Maki A, Kovaci D, Dehaene-Lambertz G, Koizumi H, Bouquet F, Mehler J. 2003. Sounds and silence: an optical topography study of language recognition at birth. Proc Natl Acad Sci U S A. 100:11702.

Petrova A, Mehta R. 2010. Regional tissue oxygenation in association with duration of hypoxaemia and haemodynamic variability in preterm neonates. Arch Dis Child Fetal Neonatal Ed. 95:F213-219.

Pogue BW, Paulsen KD, Abele C, Kaufman H. 2000. Calibration of nearinfrared frequency-domain tissue spectroscopy for absolute absorption coefficient quantitation in neonatal head-simulating phantoms. J Biomed Opt. 5:185-193.

Roche-Labarbe N, Carp SA, Surova A, Patel M, Boas DA, Grant PE, Franceschini MA. 2010. Noninvasive optical measures of CBV, StO2, CBF index, and rCMRO2 in human premature neonates' brains in the first six weeks of life. Hum Brain Mapp. 31:341-352. 
Roche-Labarbe N, Fenoglio A, Aggarwal A, Surova A, Dehaes M, Carp SA, Franceschini MA, Grant PE. 2011. Near infrared spectroscopy assessment of cerebral oxygen metabolism in the developing premature brain. J Cereb Blood Flow Metab. doi:10.1038. (accepted).

Rodriguez G, Warkentin S, Risberg J, Rosadini G. 1988. Sex differences in regional cerebral blood flow. J Cereb Blood Flow Metab. 8:783-789.

Schade J, Van Groenigen B. 1961. Structural organization of the human cerebral cortex. 1. Maturation of the middle frontal gyrus. Acta Anat (Basel). 47:74-111.

Simonds RJ, Scheibel AB. 1989. The postnatal development of the motor speech area: a preliminary study. Brain Lang. 37:42-58.

Sperry R. 1984. Consciousness, personal identity and the divided brain. Neuropsychologia. 22:661-673.

Sun T, Collura RV, Ruvolo M, Walsh CA. 2006. Genomic and evolutionary analyses of asymmetrically expressed genes in human fetal left and right cerebral cortex. Cereb Cortex. 16:i18-i25.

Sun T, Patoine C, Abu-Khalil A, Visvader J, Sum E, Cherry TJ, Orkin SH, Geschwind DH, Walsh CA. 2005. Early asymmetry of gene transcription in embryonic human left and right cerebral cortex. Science. 308:1794-1798.

Takahashi T, Shirane R, Sato S, Yoshimoto T. 1999. Developmental changes of cerebral blood flow and oxygen metabolism in children. AJNR Am J Neuroradiol. 20:917-922.

Toga AW, Thompson PM. 2003. Mapping brain asymmetry. Nat Rev Neurosci. 4:37-48.

Tzarouchi L, Astrakas L, Xydis V, Zikou A, Kosta P, Drougia A, Andronikou S, Argyropoulou M. 2009. Age-related grey matter changes in preterm infants: an MRI study. Neuroimage. 47:1148-1153.

Utsunomiya H, Takano K, Okazaki M, Mitsudome A. 1999. Development of the temporal lobe in infants and children: analysis by MR-based volumetry. AJNR Am J Neuroradiol. 20:717-723.
Van Essen DC. 2005. A population-average, landmark-and surface-based (PALS) atlas of human cerebral cortex. Neuroimage. 28:635-662.

Verhagen EA, ter Horst HJ, Keating P, Martijn A, Van Braeckel KNJA Bos AF. 2010. Cerebral oxygenation in preterm infants with germinal matrix-intraventricular hemorrhages. Stroke. 41:2901-2907.

Wolf M, Greisen G. 2009. Advances in near-infrared spectroscopy to study the brain of the preterm and term neonate. Clin Perinatol. 36:807-834.

Younkin D, Delivoria-Papadopoulos M, Reivich M, Jaggi J. 1988 Regional variations in human newborn cerebral blood flow. J Pediatr. 112:104-108.

Yu G, Durduran T, Zhou C, Wang HW, Putt ME, Saunders HM, Sehgal CM, Glatstein E, Yodh AG, Busch TM. 2005. Noninvasive monitoring of murine tumor blood flow during and after photodynamic therapy provides early assessment of therapeutic efficacy. Clin Cancer Res. 11:3543-3552.

Yu G, Floyd TF, Durduran T, Zhou C, Wang J, Detre JA, Yodh AG. 2007. Validation of diffuse correlation spectroscopy for muscle blood flow with concurrent arterial spin labeled perfusion MRI. Opt Express. 15:1064-1075.

Zeanah CH, Stafford B, Boris NW, Scheeringa M. 2008. Infant development: the first 3 years of life. In: Tasman A, Kay J, Lieberman JA, First MB, Maj M. Psychiatry. 3rd ed. Chichester (UK): John Wiley \& Sons, Ltd.

Zhao J, Ding HS, Hou XL, Zhou CL, Chance B. 2005. In vivo determination of the optical properties of infant brain using frequency-domain near-infrared spectroscopy. J Biomed Opt. 10:024028.

Zhou C, Eucker SA, Durduran T, Yu G, Ralston J, Friess SH, Ichord RN, Margulies SS, Yodh AG. 2009. Diffuse optical monitoring of hemodynamic changes in piglet brain with closed head injury. J Biomed Opt. 14:034015. 